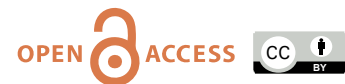

doi: $10.31729 /$ jnma.5210

\title{
Prevalence of Helicobacter Pylori in Endoscopic Gastric Biopsies of Chronic Gastritis Patients at A Tertiary Care Centre
}

\author{
Archana Tiwari, ${ }^{1}$ Ramji Rai, ${ }^{1}$ Prahar Dahal, ${ }^{1}$ Sudeep Regmi ${ }^{2}$ \\ 'Department of Pathology, Lumbini Medical College and Teaching Hospital, Tansen, Palpa, Nepal, ${ }^{2}$ Department of \\ Pathology, Manipal Medical College and Teaching Hospital, Pokhara, Nepal.
}

\section{ABSTRACT}

Introduction: Presence and severity of Helicobacter pylori (H. pylori) colonization is an important factor to decide the treatment of chronic gastritis. This study aimed to find the prevalence of $\mathrm{H}$. pylori colonization in chronic gastritis patients.

Methods: This descriptive cross-sectional study was carried out at the tertiary care center in the the western region of Nepal among the dyspeptic patients undergoing endoscopic gastric biopsy from October 2018 to March 2020 after approval from the Institutional review committee (reference number: IRC LMC 12-H/018). Convenience sampling was done to reach the sample size. Two hundred fifty cases were included in the study. Data were recorded in proforma and Data analysis was done in the statistical package for social sciences (SPSS 16.0). The severity of H.pylori colonization and gastritis was graded using the Updated Sydney System.

Results: All cases showed chronic gastritis on histopathology. H. pylori were seen in 150 (60\%) of cases. Mild, moderate, and severe H. pylori infection was seen in 59, 78, and 13 cases respectively. Out of 59 mild $H$. pylori cases, 35 (59.32\%) had mild chronic inflammation; out of 78 moderate $H$. pylori cases 51 (65.38\%) had moderate chronic inflammation and among 13 severe H. pylori cases, 10 $(76.92 \%)$ had severe chronic inflammation.

Conclusions: Prevalence of $H$. pylori colonization is high in chronic gastritis and there is a parallel increase in the severity of gastritis with an increase in the severity of $H$. pylori load.

Keywords: endoscopy; gastritis; H. pylori.

\section{INTRODUCTION}

Helicobacter pylori (H. pylori) is a bacterium, first discovered in 1982 by Robin Warren and Barry Marshall. ${ }^{1}$ Since its discovery, it has been associated with a wide spectrum of gastro-duodenal diseases including gastritis, gastro-duodenal ulcers, mucosa associated lymphoid lymphoma and gastric adenocarcinoma. ${ }^{2} \mathrm{H}$. pylori is the most common human infection of the stomach and it is also common in Nepal. ${ }^{3,4}$ The prevalence and histopathology of $\mathrm{H}$. pylori induced gastritis has been studied globally.
Gastritis is a common health problem among the Nepalese population and endoscopy along with gastric biopsies are frequently performed at our institution. Data regarding the prevalence of $H$. pylori and

Correspondence: Dr. Archana Tiwari, Department of Pathology, Lumbini Medical College and Teaching Hospital,Tansen, Palpa,Nepal.Email:archana445@gmail.com,Phone:+977-9851141238. 
histomorphology of chronic gastritis in relation to $\mathrm{H}$. pylori infection is sparse in our country.

This study aimed to find the prevalence of $\mathrm{H}$. pylori colonization in chronic gastritis patients.

\section{METHODS}

This descriptive cross-sectional study was conducted at the Department of Pathology, Lumbini Medical College, and Teaching Hospital from October 2018 to March 2020 over eighteen months. This study was approved by the Institutional Review Committee (IRC No. IRC LMC 12-H/018) of LMCTH, Tansen, Palpa, Nepal. Both the verbal and written informed consents were taken from each of the participants (or their guardians). The study population is patients who have undergone upper gastrointestinal (UGI) endoscopic evaluation. Patients with dyspeptic symptoms who underwent UGI endoscopy and gastric biopsy were included in the study. Biopsies of patients who were receiving or had received $\mathrm{H}$. pylori eradication treatment within one month and neoplastic cases were not included in the study. Biopsies of patients of all ages and both sexes were included in the study. Age and sex of the patients, detailed clinical history, and upper gastrointestinal endoscopy findings were obtained from the requisition form.

Convenience sampling was done and the minimum sample size was calculated using the formula,

$\mathrm{n}=\mathrm{Z}^{2} \times \mathrm{p} \times \mathrm{q} / \mathrm{d}^{2}$

$=(1.96)^{2} \times 0.38 \times(1-0.38) /(0.1)^{2}$

$=91$

Where,

$\mathrm{Z}=1.96$ at $95 \% \mathrm{Cl}$.

$\mathrm{p}=$ prevalence of $\mathrm{H}$. pylori infection (Miftahussurur et al 4$), 38.4 \%$

$\mathrm{q}=1-\mathrm{p}$

$d=$ margin of error, $10 \%$

The minimum sample size was calculated to be 91 .

Histopathological diagnosis was made based on antral biopsy sections findings, as an antral biopsy specimen was received in all the cases and considering the fact that in subjects with intact acid secretion, H. pylori, in particular, colonizes the gastric antrum. Biopsy specimens were fixed in $10 \%$ formalin routinely processed and paraffin blocks were sectioned at 3-4 $\mu \mathrm{m}$ thickness. Two set sections of each specimen were made and both the sections were routinely stained with hematoxylin and eosin ( $H$ \& $E$ ) and Giemsa stain respectively. Both sections from each specimen were evaluated under light microscopy by a single pathologist. The histomorphology of sections was studied on routine
H \& E stained sections. Giemsa stained sections were studied for the identification of $\mathrm{H}$. pylori.

The presence of the morphological variables $(\mathrm{H}$. pylori density; neutrophilic activity; mononuclear cell (MNC) infiltrations) was determined using the semi-quantitative method of scoring with a Visual Analogue Scale according to the updated Sydney System Classification of chronic gastritis, ${ }^{5}$ and scored 0-3 ( 0- none; 1 - mild; 2- moderate and 3- marked) to each morphological variable. Intestinal metaplasia, glandular atrophy was not graded and was simply recorded as present or absent.

Selection bias and interpretation bias was minimized as possible. Data were recorded in the proforma form. The data was then coded and entry was done in the Statistical Package for the Social Sciences (SPSS) version 16.0. The data was processed and analyzed by using simple descriptive statistics; in terms of percentage and frequency.

\section{RESULTS}

A total of 250 gastric biopsy specimens, that met the inclusion criteria were studied. On histological examination, $\mathrm{H}$. pylori were detected in 150 (60\%) patients and 100 (40\%) of patients were $\mathrm{H}$. pylori negative.

The average age of the total population was 43.43 \pm 15.13 years with age ranging $12-81$ years. One hundred sixty (64\%) samples were obtained from males and $90(36 \%)$ samples were from females, with male to female ratio $1.77: 1$. The average age of male patients was $44.19 \pm 15.38$ years, and the average age of women was $42.08 \pm 14.66$ years. The mean age of patients in $\mathrm{H}$. pylori positive group was $44.03 \pm 15.22$ ) years and mean age in $\mathrm{H}$. pylori negative group was $42.52 \pm 15.01$ years. Male to female ratio in $\mathrm{H}$. pylori positive and $H$. pylori negative group was $1.88: 1$ vs 1.63:1 respectively.

Chronic inflammation (Mononuclear cell infiltrations) was found in all of the cases of both $\mathrm{H}$. pylori positive and negative cases. The severity of chronic inflammation was seen high among $\mathrm{H}$. pylori positive cases. Moderate inflammation predominated in $\mathrm{H}$. pylori positive group and was found in 75 (50\%) cases. In $\mathrm{H}$. pylori negative group, mild inflammation predominated and was found in $51(51 \%)$ cases. In $\mathrm{H}$. pylori positive samples, Neutrophilic activity was not seen in $14(9.33 \%)$ samples, in the remaining 136 $(90.66 \%)$ specimens, neutrophilic activity was present. In $\mathrm{H}$. pylori negative samples, neutrophilic activity was not seen in $78(78 \%)$ samples, while the remaining 22 
(22\%) cases showed neutrophilic activity. Distribution of histomorphological features of gastritis among $\mathrm{H}$. Pylori positive and negative groups is shown (Table 1 ).

Table 1. Showing the distribution of histomorphological features among $\mathbf{H}$. Pylori positive and negative groups.

\begin{tabular}{|c|c|c|c|c|c|c|}
\hline \multirow[t]{2}{*}{$\begin{array}{l}\text { H. pylori } \\
\text { status (n) }\end{array}$} & \multicolumn{3}{|c|}{$\begin{array}{l}\text { Chronic inflammation (MNC } \\
\text { infiltration) } n(\%)\end{array}$} & \multirow{2}{*}{$\begin{array}{l}\text { Neutrophilic } \\
\text { activity present } n(\%)\end{array}$} & \multirow{2}{*}{$\begin{array}{l}\text { Intestinal } \\
\text { metaplasia n } \\
\text { (\%) }\end{array}$} & \multirow[t]{2}{*}{$\begin{array}{l}\text { Glandular } \\
\text { atrophy n (\%) }\end{array}$} \\
\hline & Mild & Moderate & Marked & & & \\
\hline Positive (150) & $54(36 \%)$ & $75(50 \%)$ & $21(14 \%)$ & 136 (90.66\%) & $7(4.6 \%)$ & $4(2.6 \%)$ \\
\hline Negative (100) & $51(51 \%)$ & $46(46 \%)$ & $3(3 \%)$ & $22(22 \%)$ & $0(0 \%)$ & $0(0 \%)$ \\
\hline
\end{tabular}

Out of $150 \mathrm{H}$. pylori positive cases, mild $\mathrm{H}$. pylori colonization was seen in 59 (39.3\%) samples, moderate colonization was seen in 78 (52\%), and marked colonization was seen in $13(8.7 \%)$ specimens. Of 59 mild $\mathrm{H}$. pylori gastritis, mild chronic inflammation predominated and was found in 35 (59.32\%) cases. Among 78 moderate $\mathrm{H}$. pylori positive group, moderate chronic inflammation predominated and was found in $51(65.38 \%)$ cases. Of 13 marked grade of $\mathrm{H}$. pylori positive group, marked inflammation predominated and was found in $10(76.92 \%)$ cases. Distribution of different grades of chronic inflammation in different severity of $\mathrm{H}$. pylori colonization is shown in (Table 2).

Table 2. Distribution of different grades of chronic inflammation in different severity of $\mathrm{H}$. pylori colonization.

The density of $\mathrm{H}$. Chronic inflammation (Mononuclear cell infiltrations) $\mathbf{n}(\%)$

pylori

Mild

Mild

Moderate

$35(59.32 \%)$

Marked
Moderate

21 (35.59\%)

51 (65.38\%)

3 (23.07\%)

\section{Marked}

$3(5.08 \%)$

$8(10.25 \%)$

$10(76.92 \%)$
Total n (\%)

$59(100 \%)$

$78(100 \%)$

$13(100 \%)$
Among 59 mild $\mathrm{H}$. pylori positive group, mild neutrophilic activity predominated and was seen in 29 $(49.15 \%)$ cases. Out of 78 moderate H. pylori positive group, moderate neutrophilic activity predominated and was found in $39(50 \%)$ cases. Out of 13 marked $\mathrm{H}$. pylori positive group, marked neutrophilic activity predominated and was found in $9(69.23 \%)$. Distribution of different grades of neutophilic activity in different severity of $\mathrm{H}$. pylori colonization is shown (Table 3 ).

Table 3. Distribution of different grades of neutophilic activity in different severity of $\mathbf{H}$. pylori colonization.

\begin{tabular}{|c|c|c|c|c|c|}
\hline \multirow{2}{*}{$\begin{array}{l}\text { Density of H. } \\
\text { pylori }\end{array}$} & \multirow[b]{2}{*}{ No } & \multicolumn{2}{|c|}{ Neutrophilic activity $\mathbf{n}(\%)$} & \multirow[b]{2}{*}{ Marked } & \multirow{2}{*}{ Total n (\%) } \\
\hline & & Mild & Moderate & & \\
\hline Mild & $10(16.94 \%)$ & $29(49.15 \%)$ & $19(32.2 \%)$ & $1(1.69 \%)$ & $59(100 \%)$ \\
\hline Moderate & $4(5.12 \%)$ & $24(30.76 \%)$ & $39(50 \%)$ & $11(14.1 \%)$ & $78(100 \%)$ \\
\hline Marked & $0(0 \%)$ & $1(7.69 \%)$ & $3(23.07 \%)$ & $9(69.23 \%)$ & $13(100 \%)$ \\
\hline
\end{tabular}

\section{DISCUSSION}

In this study, histopathological reporting was done in all cases on antral biopsies, as antrum was the most common site from which biopsies were received. For the consideration of the patient's comfort and operator's convenience, it was difficult to follow the extensive endoscopy biopsy protocol of five biopsy samples as per the updated Sydney system, from every patient. Eriksson et al. have recommended that antrum is the most likely site of histopathological findings in gastritis. ${ }^{6}$ Similar to our study, Garg et al, ${ }^{7}$ Park et al, ${ }^{8}$ and Dhakhwa et al, ${ }^{9}$ have also considered their studies mainly on antral biopsies. 
In our study, the age group affected by the varying grade of chronic Gastritis ranged from 12-81 years and the mean age was 43.43 years. This finding is similar to findings of other studies where the mean age was 47 years. 7,10

A predominance of a male having chronic inflammation has been noticed in this study with an M:F ratio of 1.77:1 which is similar to the study of Garg et al, Park et al, Chen et al, and Pruthi et al, where they reported an $\mathrm{M}: \mathrm{F}$ ratio of $2.1: 1,2.8: 1,1.8: 1,2.3: 1$ and respectively. ${ }^{7,8,11,12} \mathrm{H}$.pylori infection was more common in males than females, which is at par with the result of many previous studies. $7,8,12,13$ However, this finding is contrary to the study by Maharjan et al, where females were found to have a higher rate of $\mathrm{H}$. pylori infection. ${ }^{14}$

In this study, $\mathrm{H}$. pylori infection was identified in 150 $(60 \%)$ cases out of $250(100 \%)$ specimens included in the study. Similar figures are seen in the study conducted by Park et al. ${ }^{8}$ In previous studies, the prevalence of $\mathrm{H}$. pylori varied from $43.66 \%$ of Garg et al to $93.7 \%$ of Hassan et al. ${ }^{7,10}$

This variation in the prevalence of $\mathrm{H}$. pylori may be due variation in biopsy sampling sites for varying intragastric colonization, host immune response, level of acid production, and prior treatment with proton pump inhibitors or anti $\mathrm{H}$. pylori antimicrobial agents. Multiple site sampling may be needed to improve results.

Chronic Gastritis (Mononuclear cell infiltration) was seen in $250(100 \%)$ cases in the present study. Various other studies have shown findings similar to this study. ${ }^{7,8,15}$ Majority of inflammation in this study were of moderate grade $(48.8 \%)$ followed by mild $42 \%$ and marked $9.6 \%$, which is similar to the other studies. ${ }^{8,14,15}$ In contrary to this, a study by Garg et al. found that majority of inflammation was mild. ${ }^{7}$ Moreover, H. pylori was detected in 150 (60\%) cases of Chronic gastritis that is consistent with a previous study. ${ }^{14}$

In this study, we have seen the severity of chronic inflammation parallels the severity of $H$. pylori density. Mild chronic inflammation predominated in mild $\mathrm{H}$. pylori group. Moderate chronic inflammation predominated inthe moderate $\mathrm{H}$. pylori group. Severe chronic inflammation predominated in the severe $\mathrm{H}$. pylori group. This is at par with the results of other studies, ${ }^{7,15-19}$, however, it is contrary with the study of Park et al, as they found no association between the grade of mononuclear cell infiltration and density of H. pylori. ${ }^{8}$

Overall, Neutrophilic activity was seen in 158 (63.2\%) cases out of a total of $250(100 \%)$ cases. This is lower than the studies by Park et al. and A Hussein et al; where neutrophilic activity was seen in $78.75 \%$ and $84 \%$ cases respectively. 8,15 However, the result in the present study out numbered the observation of previous studies by Garg et al $(33.33 \%)$, Hassan et al (31.84\%), and Maharjan et al (33.6\%). ${ }^{7,10,14}$

In $H$. pylori negative group (100 cases), neutrophilic activity was seen only in $22(22 \%)$. In $\mathrm{H}$. pylori positive group (150 cases), neutrophilic activity was seen only in 136 (90.66\%) cases. In previous studies, the presence of neutrophilic activity in $\mathrm{H}$. pylori positive cases was seen from low to as high as $100 \%$ cases. The neutrophilic activity was present in $100 \% \mathrm{H}$. pylori positive cases in a study by $A$ Hussein et al. and Dhakhwa et.al. ${ }^{9,15}$ In the study conducted by Maharjan et al, ${ }^{14}$ Neutrophilic activity was seen in $(40.7 \%)$ of $\mathrm{H}$. pylori positive cases.

Similar to chronic inflammation, the severity of neutrophilic activity parallels the severity of $\mathrm{H}$. pylori density. Mild neutrophilic activity predominated in a mild $H$. pylori group. Moderate neutrophilic activity predominated in moderate $H$. pylori group. Severe neutrophilic activity predominated in the severe $\mathrm{H}$. pylori group. This finding is similar to previous other studies. ${ }^{7,14-17}$ However, Park et al. ${ }^{8}$ observed nostatistical association between neutrophilic activity and $\mathrm{H}$. pylori concentration.

In $\mathrm{H}$. pylori positive cases, glandular atrophy was seen in $4(2.6 \%)$ and intestinal metaplasia was seen in 7 (4.6 $\%)$ cases in the present study. No cases of glandular atrophy or intestinal metaplasia were seen in $\mathrm{H}$. pylori negative cases. In contrary to this finding Dakhwa et al, Pruthi et al, and Maharjan et al found glandular atrophy and intestinal metaplasia in both $\mathrm{H}$. pylori negative and positive cases..$^{9,12,14}$

The limitation of this study is that only antral biopsy was considered for this study and the updated Sydney system was not strictly followed regarding sampling of gastric sites. Adherence to updated guidelines of biopsy sites, considering the topographic distribution of $\mathrm{H}$. Pylori, the diagnostic yield of gastric inflammatory conditions and $\mathrm{H}$. pylori detection would have improved.

\section{CONCLUSIONS}

This study concludes that the prevalence of $\mathrm{H}$. pylori infection is high in chronic gastritis. There is a parallel increase in the severity of chronic inflammation (lymphoplasmacytic infiltration) and neutrophilic activity as the severity of $\mathrm{H}$. pylori infection increases. Greater the density of $H$. pylori, the larger is the degrees of chronic inflammation and neutrophilic 
Tiwari et al. Prevalence of Helicobacter Pylori in Endoscopic Gastric Biopsies of Chronic Gastritis Patients at a Tertiary Care Centre

activity. Histomorphological search for $\mathrm{H}$. pylori should be meticulously initiated if neutrophils are seen in the foveolar epithelium surface or glandular neck. Grades of H. pylori colonization can further guide chemotherapy regime, duration, and follow up to eradicate $\mathrm{H}$. pylori and prevent complications related to it. Moreover, we also recommend to include multiple sites sampling protocol as far as possible to avoid false negative results.

\section{Conflict of Interest: None.}

\section{REFERENCES}

1. R Warren, B Marshall. Unidentified Curved Bacilli on Gastric Epithelium in Active Chronic Gastritis. Lancet. 1983 Jun;1(8336):1273-5. [PubMed]

2. JQ Huang, S Sridhar, Y Chen, R H Hunt. Meta-analysis of the relationship between Helicobacter pylori seropositivity and gastric cancer. Gastroenterology. 1998 Jun;114(6):1169-79. [PubMed]

3. Barik A Salih. Helicobacter pylori Infection in Developing Countries: The Burden for How Long? Saudi J Gastroenterol. 2009 Jul;15(3):201-7. [ubMed]

4. Miftahussurur M, Sharma RP, Shrestha PK, Suzuki R, Uchida T, Yamaoka Y. Molecular Epidemiology of Helicobacter pylori Infection in Nepal: Specific Ancestor Root. PLOS ONE. 2015 Jul 30;10(7):e0134216. [Full Text]

5. F Dixon, R M Genta, J H Yardley, P Correa. Classification and Grading of Gastritis: The Updated Sydney System. Am J Surg Pathol. 1996 Oct;20(10):1161-81. [PubMed]

6. M N K Eriksson, M A Färkkilä, M E Voutilainen, P E Arkkila. The clinical value of taking routine biopsies from the incisura angularis during gastroscopy. Endoscopy. 2005 Jun;37(6):532-6. [PubMed]

7. Garg B, Sandhu V, Sood N, Sood A, Malhotra V. Histopathological analysis of chronic gastritis and correlation of pathological features with each other and with endoscopic findings. Pol J Pathol. 2012;3:172-8. [PubMed]

8. Park JW, Kim MK, Park SM. Influence of Helicobacter pylori Colonization on Histological Grading of Chronic Gastritis in Korean Patients with peptic Ulcer. Korean J Intern Med. 1995 Jul 31;10(2):125-9. [PubMed | Full Text]

9. Dhakhwa R, Acharya IL, Shrestha HG, Joshi DM, Lama S, Lakhey M. Histopathologic study of chronic antral gastritis. J Nepal Health Res Counc. 2012 Jan;10(1):57-60. [PubMed]

10. Hassan TMM, Al-Najjar SI, Al-Zahrani IH, Alanazi FIB, Alotibi MG. Helicobacter pylori chronic gastritis updated Sydney grading in relation to endoscopic findings and H. pylori IgG antibody: diagnostic methods. J Microsc Ultrastruct. 2016 Dec 1;4(4):167-74. [PubMed]
11. Chen XY, Liu WZ, Shi Y, Zhang DZ, Xiao SD, Tytgat GNJ. Helicobacter pylori associated gastric diseases and lymphoid tissue hyperplasia in gastric antral mucosa. J Clin Pathol. 2002 Feb 1;55(2):133-7. [PubMed]

12. Pruthi S, Nirupama M, Chakraborti S. Evaluation of gastric biopsies in chronic gastritis: Grading of inflammation by Visual Analogue Scale. Med J Dr Patil Univ. 2014 Jul 1;7(4):463. [Full Text]

13. Udoh MO, Obaseki DE. Histopathological evaluation of $\mathrm{H}$. pylori associated gastric lesions in Benin city, Nigeria. East Afr Med J. 2012;89(12):408-413. [Full Text]

14. Maharjan S. Helicobacter Pylori Associated Chronic Gastritis and Application of Visual Analogue Scale for the Grading of the Histological Parameters in Nepal. Biomed J Sci \& Tech Res .2017 May 25;1(1):28-34. [Full Text]

15. A. Hussein H. Evaluation of Chronic Gastritis in Endoscopic Antral Biopsies Using the Up-dated Sydney System. Ann Coll Med Mosul. 2020 Jan 29;41(2):95-105. [Full Text]

16. Ghasemi Basir HR, Ghobakhlou M, Akbari P, Dehghan A, Seif Rabiei MA. Correlation between the Intensity of Helicobacter pylori Colonization and Severity of Gastritis [Internet]. Gastroenterology Research and Practice. 2017;e8320496. [Full Text]

17. Correa G S, Cardona A AF, Correa G T, Correa L LA, García G HI, Estrada M S. Prevalence of Helicobacter pylori and Histopathological Features in Gastric Biopsies from Patients with Dyspeptic Symptoms at a Referral Center in Medellin. Rev Colomb Gastroenterol. 2016 Jan;31(1):9-15. [Full Text]

18. Yakoob MY, Hussainy AS. Chronic gastritis and Helicobacter pylori: a histopathological study of gastric mucosal biopsies. J Coll Physicians Surg Pak. 2010;20(11):773-5. [PubMed]

19. Choi WR, Song $\mathrm{CH}$, Sheen $\mathrm{DH}$, Yang SS, Lim SH, Lee $\mathrm{KH}$, et al. Difference in the Distribution Pattern of Helicobacter pylori and Grade of Gastritis in the Antrum and in the Body between Duodenal Ulcer and Benign Gastric Ulcer Patients. Korean J Intern Med. 2000 Jan 31;15(1):32-6. [Full Text] 